\title{
FDTD and Single Scattering Formulation for Simulation of Foliage Camouflaged Hard Targets
}

\author{
Mojtaba Dehmollaian, Hossein Mosallaei, and Kamal Sarabandi \\ Radiation Laboratory \\ Department of Electrical Engineering and Computer Science \\ The University of Michigan, Ann Arbor, MI 48109-2122 \\ E-mail: saraband@eecs.umich.edu
}

\begin{abstract}
In this paper an electromagnetic scattering model is presented that allows simulation of radar response from hard targets embedded within a foliage canopy. The modeling approach is based on a hybrid foliage propagation and scattering method and a full-wave simulation for a finitesize target. The hybrid model allows for inclusion of effects of foliage attenuation and scattering in addition to near-filed foliage-target and targetfoliage interactions. The purpose of developing this model is to investigate the effect of foliage on target signature and determine methodologies that would enhance target detection.
\end{abstract}

\section{INTRODUCTION}

For detection and identification of a hard target inside a forest an accurate model is needed. Characterization of electromagnetic (EM) scattering from camouflaged complex targets in an exact manner, is a very challenging problem. This problem consists of two parts, 1) description of propagation through and scattering from the forest, and 2) calculation of scattering from the hard targets, illuminated by the field inside the forest. In this paper we propose a hybrid model that accurately calculates the backscatter field from embedded targets. The proposed hybrid model is composed of three parts, foliage model, FDTD full-wave analysis, and calculation of backscattered field using reciprocity. The foliage model is a coherent single scattering model that preserves the geometry of tree structures using a statistical Lindermayer system. In this model, tree constituents are modeled by dielectric cylinders and disks, whose scattering are analytically derived using high- and low-frequency techniques. Scattering models are enhanced so that scattered field computation is possible in the near-field region of the scatterers [1]. The mean field is computed using Foldy's approximation, which accounts for the phase change as well as extinction due to the scattering and absorption of the tree particles. The realistic foliage structure is placed above a dielectric half space, representing the ground plane. The mean field and its reflection from the ground are used as the excitation fields for every scatterer. Monte Carlo simulation is used to generate many realizations of the forest trees from which the statistics of the scattered fields are derived. The scattered field from all tree components and the mean fields are used as the excitation for the hard target. A fictitious Huygens' surface is used to enclose the target. The magnitude and phase of the forest field over this surface is calculated and used to illuminate the hard target. An FDTD code is implemented to compute the scattered field over the Huygens' surface. Reciprocity is finally applied to compute the scattered field from the target and its interaction with the surrounding foliage.

\section{FORMULATION}

EM scattering from hard targets inside foliage, with arbitrary shape can be carried out using a number of approximation and numerical approaches. Since the wavelength is comparable to the typical hard target dimensions, such as 
ground vehicles, in VHF frequency band (20$200 \mathrm{MHz}$ ), high frequency approximation techniques are not usually applicable. Therefore, exact numerical approaches such as MoM or FDTD are appropriate to treat the scattering problem. In this paper, we used FDTD technique, to calculate the scattering from a hard target located inside a virtual rectangular box (Huygens' surface). Using the forest model, the field on the box is computed and considered as the incident field for the target. Since FDTD approach is a time domain analysis the frequency domain field must be transferred to the time domain representation $\bar{\varepsilon}_{1}(t)$, given by

$$
\bar{\varepsilon}_{1}(t)=2 \operatorname{Real}\left\{\int_{f_{\text {start }}}^{f_{\text {stop }}} \bar{F}_{1} e^{-i 2 \pi f t} d f\right\}
$$

where $\bar{F}_{1}$ can be $\bar{E}_{1}$ or $\bar{H}_{1}$, respectively for the electric and magnetic fields computed inside the foliage, using the forest model. Depending on the desired frequency range of simulation the complex total field quantities from $f_{\text {start }}$ to $f_{\text {stop }}$ are computed over the FDTD box at $\mathrm{N}$ frequency points. The choice of $\mathrm{N}$ (increments in frequency) depends on the size of the FDTD box or equivalently the alias-free time domain period. Using FDTD full-wave analysis the scattered field by the target is calculated at the same grid points, and transferred to frequency domain, using

$$
\bar{F}_{2}=\int_{-\infty}^{+\infty} \bar{\varepsilon}_{2}(t) e^{+i 2 \pi f t} d t
$$

According to Huygens' principle, tangential electric and magnetic scattered fields on a closed surface are enough to express the scattered field every where, having the Green's function of the medium. In complex random media such as a forest canopy the exact computation of the Green's function is not possible. However we can solve the problem using the reciprocity theorem. Based on reciprocity theorem for two sets of sources and fields denoted by subscripts 1 and 2, we have [3]

$$
\begin{aligned}
& \int_{V_{2}}\left(\bar{E}_{1} \cdot \bar{J}_{2}-\bar{H}_{1} \cdot \bar{M}_{2}\right) d \mathbf{r}= \\
& \int_{V_{1}}\left(\bar{E}_{2} \cdot \bar{J}_{1}-\bar{H}_{2} \cdot \bar{M}_{1}\right) d \mathbf{r}
\end{aligned}
$$

We assume that $\bar{M}_{1}=0$ and $\bar{J}_{1}$ is an elementary current at the radar position, given by

$$
\bar{J}_{1}=\frac{4 \pi}{i k_{o} Z_{o}} \frac{r_{R}}{e^{i k_{o} r_{R}}} \delta\left(\bar{r}-\bar{r}_{R}\right)(-\hat{p})
$$

where $k_{o}$ and $Z_{o}$ are the propagation constant and characteristic impedance of free space, respectively. The unit vector $\hat{p}$ can be along principal polarization vectors $\hat{h}_{i}$ or $\hat{v}_{i}$. Denoting $\bar{J}_{2}$ and $\bar{M}_{2}$ as the electric and magnetic scattering sources on the FDTD surface enclosing the target, the far-field backscattering from a target, $\bar{E}_{2}$, can be derived by substituting (4) in (3). Therefore we have

$$
\begin{aligned}
& \bar{E}_{2} \cdot(-\hat{p})=\frac{i k_{o} Z_{o}}{4 \pi} \frac{e^{i k_{o} r_{R}}}{r_{R}} \\
& \int_{V_{2}}\left(\bar{E}_{1} \cdot \bar{J}_{2}-\bar{H}_{1} \cdot \bar{M}_{2}\right) d \mathbf{r}
\end{aligned}
$$

The equivalent electric and magnetic current sources on the Huygens' surface are simply expressed by $\bar{J}_{2}(\bar{r})=\hat{n} \times \bar{H}_{2}(\bar{r})$ and $\bar{M}_{2}(\bar{r})=$ $-\hat{n} \times \bar{E}_{2}(\bar{r})$ where $\hat{n}$ is the normal unit vector pointing outward the Huygens' surface. Using (5) it can be shown that each component of the scattering matrix can be computed from

$$
\begin{aligned}
& \mathbf{S}_{p q}= \pm \frac{i k_{o} Z_{o}}{4 \pi} \\
& \int_{\mathbf{S}}\left[\bar{H}_{2 q} \times \bar{E}_{1 p}+\bar{E}_{2 q} \times \bar{H}_{1 p}\right] \cdot \hat{n} d s
\end{aligned}
$$

where $p$ and $q$ represent the $h$ or $v$ polarizations. The first and second subscripts of scattering elements denote the receive and transmit polarizations, respectively. The minus sign in (6) is chosen when the receive polarization is vertical. Once the incident field, computed by forest model, and the local scattered field from target, computed by FDTD are known the backscattered field can be computed from (6). Finally, the Radar Cross Section (RCS) of the target is calculated from,

$$
\sigma_{p q}^{s}=4 \pi\left|\mathbf{S}_{p q}\right|^{2}
$$

\section{Simulation RESUlts}

The proposed hybrid method is validated, by considering a $3 m \times 3 m$ metallic dihedral corner reflector in free space. Since the target is inside an FDTD box with size of $3.96 \mathrm{~m} \times 3.96 \mathrm{~m} \times 3.96 \mathrm{~m}$, maximum frequency increment $\Delta f=43.74 \mathrm{MHz}$ 


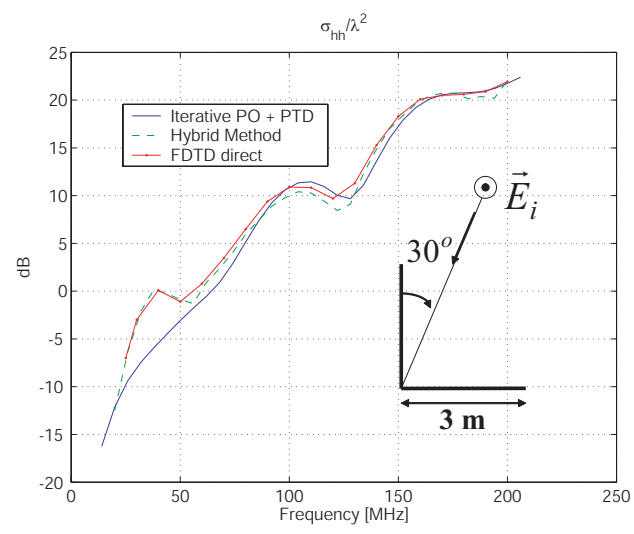

Fig. 1. Comparison of backscattering RCS from a dihedral corner reflector in free space, calculated by direct FDTD, hybrid method, and physical optics + PTD approximation for an $\mathrm{H}$-polarized incident plane wave propagating along $\theta_{i}=30^{\circ}$ and $\phi_{i}=180^{\circ}$.

is required to have alias-free time domain response. For this simulation we used $\Delta f=6 \mathrm{MHz}$. The cell size in FDTD code is set to $12 \mathrm{~cm}$. Due to the size of the FDTD box and cell size, time span of about $212 \mathrm{nsec}$ with time step of $0.83 \mathrm{nsec}$ is set for FDTD technique to provide an accurate solution. Figure 1 shows the backscatter RCS of the dihedral, using direct FDTD, the hybrid approach, and physical optics (PO) + physical theory of diffraction (PTD) approximation as a function of frequency, for an $\mathrm{H}$-polarized incident plane wave, propagating along $\theta_{i}=30^{\circ}$ and $\phi_{i}=$ $180^{\circ}$. An excellent agreement is shown. Complete simulation is considered where the dihedral is placed $0.5 \mathrm{~m}$ above a dielectric ground plane with relative permittivity of $\epsilon_{r}=5.62+i 0.94$. Eight pine trees, generated by statistical L-system, having average height of $15 \mathrm{~m}$, crown radius of $3 \mathrm{~m}$, crown height of $10 \mathrm{~m}$, trunk radius of $10 \mathrm{~cm}$, and more than 5000 branches per tree, are arranged in $144 \mathrm{~m}^{2}$ around the target. Since the entire scatterers in the forest are inside a volume with maximum dimension of about $22.65 \mathrm{~m}$, the maximum frequency increment $\Delta f=13.25 \mathrm{MHz}$ is required to have alias-free time domain response. For the same parameters as previous simulation $(\Delta f=6 \mathrm{MHz}$, cell size $=12 \mathrm{~cm}$, time span $=212$ nsec , time step $=0.83$ nsec ) simulation is performed. Figure 2 shows the backscattered RCS response of the dihedral as a function of

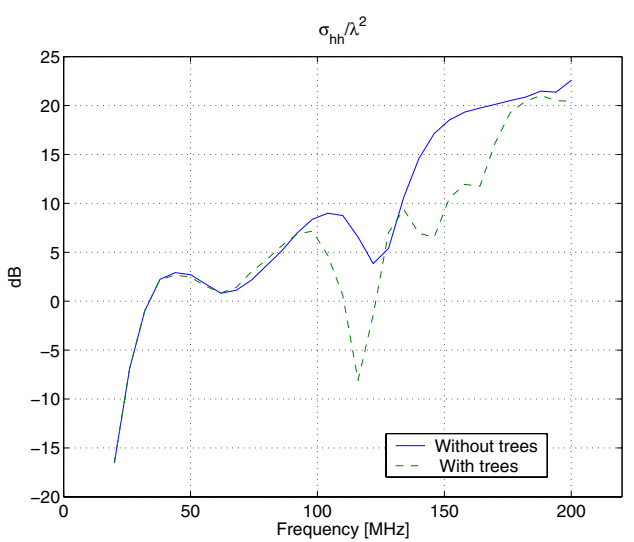

Fig. 2. Comparison of backscattering RCS from a dihedral corner reflector, above a dielectric ground plane with $\epsilon_{r}=$ $5.62+i 0.94$, calculated by the hybrid method, inside and outside of the forest for horizontal polarization of incident field propagating along $\theta_{i}=30^{\circ}$ and $\phi_{i}=180^{\circ}$.

frequency once with and once without foliage, for horizontal polarization of incident field. The backscatter responses of the target embedded in the foliage, shown in this figure, does not include the forest backscatter itself. Based on simulation results, it is observed that the target response is significantly changed for frequencies higher than $60 \mathrm{MHz}$. Clearly, the electric size of the target as well as forest parameters, such as density and number of the trees, determine this frequency.

\section{REFERENCES}

[1] Il-Suek Koh, and Kamal Sarabandi, Polarimetric Channel Characterization of Foliage for Performance Assessment of GPS Receivers Under Tree Canopies, IEEE Trans. Antennas Propagat., Vol. 50, No. 5, May 2002.

[2] H. Mosallaei and Y. Rahmat-Samii, Broadband characterization of complex periodic EBG structures: An FDTD/Prony technique based on the split-field approach, Electromag. J., vol. 23, no. 2, pp. 135-151, Feb.Mar. 2003.

[3] Roger F. Harington, Time-Harmonic Electromagnetic Fields, John Wiley and Sons, NY, 2001.

[4] Hans Israelsson, Lars M. H. Ulander, Torleif Martin, and Jan I. H. Askne, A Coherent Scattering Model to Determine Forest Backscattering in VHF-Band, IEEE Trans. Geosci. and Remote Sensing, Vol. 38, No. 1, Jan. 2000. 